\title{
Evaluation participative de types de semis et de l'écartement en culture de fonio (Digitaria exilis Stapf.) au Bénin
}

\author{
Cyrille Kanlindogbe, Msc. \\ Emmanuel Sekloka, Phd
}

Laboratoire de Phytotechnie, d'Amélioration et de Protection des Plantes,

Faculté d'Agronomie, Université de Parakou, Bénin

Richard Ayelesso, Msc.

Louvain Coopération, Bénin

Jean Opala, Msc.

Eclosio (ex Aide au Développement Gembloux), Bénin

Valérien Zinsou, Phd

Laboratoire de Phytotechnie, d'Amélioration et de Protection des Plantes,

Faculté d'Agronomie, Université de Parakou, Bénin

Armand Natta, Phd

Laboratoire d'Ecologie, de Botanique et de Biologie Végétale,

Faculté d'Agronomie, Université de Parakou, Bénin

Doi:10.19044/esj.2020.v16n3p211 ～URL:http://dx.doi.org/10.19044/esj.2020.v16n3p211

\section{Résumé}

Le fonio, ancienne céréale africaine, est négligée malgré son rôle crucial dans la sécurité alimentaire. Cette étude vise à comparer les types de semis pour l'amélioration des techniques culturales de cette espèce au Bénin. Les semis en lignes continues à $25 \mathrm{~cm}$ et $30 \mathrm{~cm}$ entre lignes, et à la volée ont été comparés dans un dispositif de bloc aléatoire complet dispersé dans dixhuit villages. Les données agronomiques ont été collectées et les perceptions paysannes recueillies au moyen de questions structurées. Les statistiques descriptives, analyses de variance et tests de Khi-deux ont été réalisées. Les semis en ligne ont consommé près du double de la quantité de semence utilisée à la volée. Ils ont nécessité plus de deux fois la durée du semis à la volée. Cependant, le désherbage a été plus facile et rapide avec les semis en lignes. Les types de semis n'ont eu aucun effet significatif sur la verse des plants. Les rendements en grain ont été meilleurs pour les semis en lignes continues avec un surplus de 150 à près de $200 \mathrm{~kg} / \mathrm{ha}$ par rapport à la volée. Les deux écartements du semis en ligne ont présenté des tendances similaires pour l'ensemble des variables évaluées. Les producteurs ont noté que le désherbage 
à la houe est facile dans les semis en ligne qu'à la volée, bien que cette opération se trouve laborieux et difficile. Le semis en ligne amélioré par un outil mécanique équipé de semoir, apporterait une plus-value à la production du fonio.

Mots Clés : Semis En Ligne, Semis À La Volée, Perceptions Paysannes, Fonio, Bénin

\title{
Participatory Evaluation of Sowing Types and Spacings in Fonio (Digitaria Exilis Stapf.) Cultivation in Benin
}

\author{
Cyrille Kanlindogbe, Msc. \\ Emmanuel Sekloka, Phd
}

Laboratoire de Phytotechnie, d'Amélioration et de Protection des Plantes,

Faculté d'Agronomie, Université de Parakou, Bénin

Richard Ayelesso, Msc.

Louvain Coopération, Bénin

Jean Opala, Msc.

Eclosio (ex Aide au Développement Gembloux), Bénin

Valérien Zinsou, Phd

Laboratoire de Phytotechnie, d'Amélioration et de Protection des Plantes,

Faculté d'Agronomie, Université de Parakou, Bénin

Armand Natta, Phd

Laboratoire d'Ecologie, de Botanique et de Biologie Végétale,

Faculté d'Agronomie, Université de Parakou, Bénin

\section{Abstract}

Fonio, former African cereal, is neglected despite its crucial role in food security. This study aims to compare types and spacings of sowing for improving fonio cultivation techniques in Benin. Sowing in continuous rows at $25 \mathrm{~cm}$ and $30 \mathrm{~cm}$ between rows, and by broadcasting were compared in dispersed random complete block design in eighteen villages. Agronomic data was collected and farmer perceptions collected through structured questionnaires. Descriptive statistics, analysis of variance and Chi-square tests were performed. Sowing in rows consumed almost double the seed rate used for broadcasting. They required more than twice the time spent for 
broadcasting sowing. However, weeding was easier and faster to manage in rows sowing. Sowing types had no significant effect on plant lodging. Grain yields were better under continuous rows sowing with surplus of 150 to nearly $200 \mathrm{~kg} / \mathrm{ha}$ over broadcasting technique. The two spacing of in-rows sowing showed similar trends for all variables assessed. Farmers noted that hoe weeding is easier in row sowing than by broadcasting, although they find difficult row seeding method. In-row sowing improved by mechanical tool equipped with a seeder, would bring an added value to fonio production chain.

Keywords: In-Row Sowing, Broadcasting, Farmers' Perceptions, Fonio, Benin

\section{Introduction}

Le fonio, l'une des premières céréales cultivées en Afrique de l'ouest, est une plante d'une grande importance pour les populations de par ses vertus médicinales, ses qualités gustatives et son intérêt pour la sécurité alimentaire (Cruz et al., 2011).

$\mathrm{Au}$ Bénin, contrairement aux autres céréales telles que le maïs, le sorgho, le mil et le riz, dont la culture se pratique un peu partout sur toute l'étendue du territoire national, le fonio (Digitaria spp.) apparaît comme une culture essentiellement locale voire endémique de l'Atacora (AdoukonouSagbadja et al., 2010 ; Dansi et al., 2010). Cette céréale présente une importance socioculturelle chez les peuples Otamari de la commune de Boukoumbé, principale productrice qui à elle seule fournit $74 \%$ de la production nationale (MAEP, 2010 ; Paraïso et al., 2011 ; Ballogoun, 2013). En dépit de ses multiples vertus et potentiels; il reste une plante longtemps marginale et négligée dans les programmes nationaux de recherche et de promotion des cultures vivrières. A l'instar de plusieurs pays de l'Afrique de l'Ouest, beaucoup de travaux de recherche ont porté sur la gestion de la récolte et de la post récolte situés en aval de la chaîne de valeur du fonio. Les autres maillons situés en amont, de la chaîne de valeur notamment les techniques culturales demeurent traditionnelles, rudimentaires et peu étudiées. Ce qui résulte en la pénibilité des opérations culturales très caractéristique du fonio avec des rendements très faibles variant généralement entre $200-700 \mathrm{~kg} / \mathrm{ha}$ (Badiane, 2006 ; Vodouhè et Achigan Dako, 2006).

L'objectif général de cette étude est d'évaluer types et écartements de semis dans un contexte d'amélioration des techniques culturales pour la relance de la production du fonio au Bénin. Plus spécifiquement, il s'agit d'évaluer l'effet de types et écartements de semis sur les paramètres agronomiques du fonio, et de recueillir les perceptions paysannes sur la pénibilité des opérations culturales. 


\section{Matériel et méthodes \\ 1.1. Milieu d'étude}

Le dispositif d'évaluation a été conduit à Boukombé principale commune de production de fonio, localisée entre $10^{\circ}$ et $10^{\circ} 40^{\prime}$ de latitude Nord et $0^{\circ} 75^{\prime}$ et $1^{\circ} 30^{\prime}$ de Longitude Est (MAEP, 2010). Boukombé se situe dans le département de l'Atacora, Nord-Ouest Bénin (Figure 1). Avant l'installation d'essais, deux assemblées villageoises ont été animées dans la commune pour présenter et expliquer aux producteurs les objectifs de la recherche et le dispositif expérimental. Dix-huit villages répartis sur quatre arrondissements de la commune ont été retenus pour la conduite du dispositif (Figure 1). Ces villages ont été choisis sur la base de l'importance de la production, l'accessibilité des sites, l'existence de groupements de producteurs du fonio et l'ouverture aux innovations agricoles.

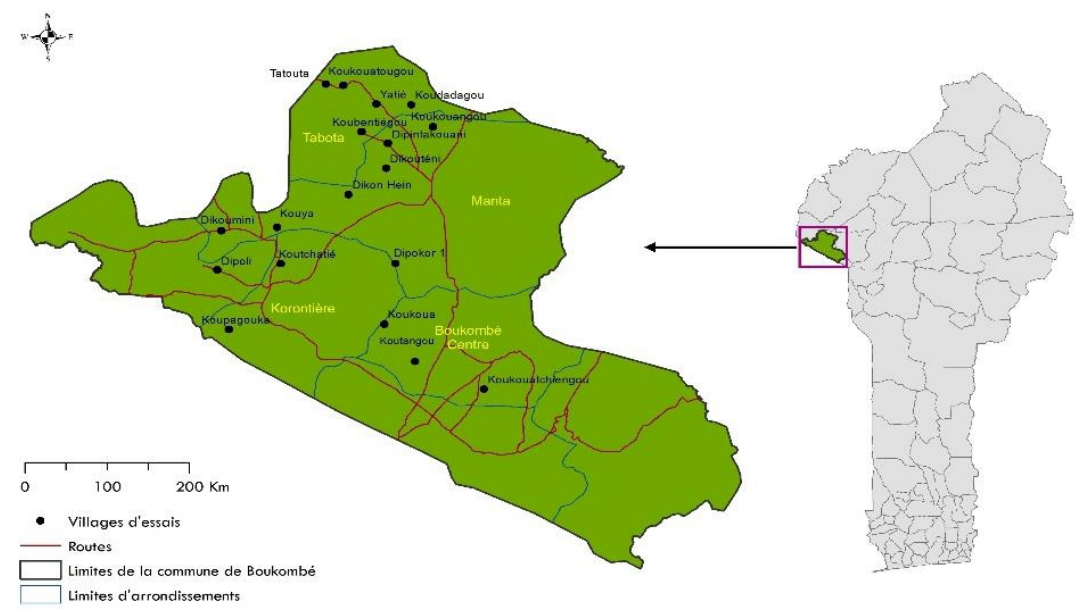

Figure 1 : Localisation des villages d'expérimentation dans la commune de Boukoumbé (Source : Données IGN, et GPS extrex)

\subsection{Dispositif expérimental}

Pour cette évaluation participative en milieu paysan, le dispositif utilisé est celui de bloc aléatoire dispersé avec 03 traitements répétés dans les dix-huit villages. Chaque village abrite ainsi une répétition. Les trois traitements comparés étaient le semis ordinaire à la volée (SOV), le semis en ligne continue avec respectivement des écartements de 25 (SLC25) et de $30 \mathrm{~cm}$ entre ligne (SLC30). Dans chaque répétition, les superficies des parcelles élémentaires étaient les mêmes. Le matériel végétal utilisé pour l'expérimentation est constitué des semences de trois écotypes de fonio disponibles et fournies par les groupements de producteurs de chaque village. Ces écotypes sont nommés 'Iporapia', 'Iporawan' et 'Dipowan' par les Otamari. 


\subsection{Installation et conduite des essais}

La préparation des parcelles (labour) a été réalisée à plat à la traction animale. Les essais ont été installés en fin juin 2017. Les différentes opérations culturales (semis, entretien et récolte) de chaque essai ont été effectuées par les membres de chaque groupement de producteurs. Les opérations d'entretiens ont consisté en un seul désherbage un mois environ après le semis. Aucune fumure, aucun pesticide, ni herbicide n'a été appliqué. A la maturation, la récolte a été réalisée par les producteurs au moyen des couteaux tranchants pour sectionner les pailles du fonio regroupées en gerbes, séchées à l'air libre, égrenées et vannées.

\subsection{Collecte des données}

Les données collectées ont porté sur les variables agronomiques, les doses de semences, les durées des opérations culturales et les perceptions paysannes sur les opérations culturales sous chaque type de semis (Tableau 2). Les traits agronomiques ont été mesurés à partir des descripteurs des céréales (riz, maïs, sorgho, mil, blé) (Bioversity International et al., 2007) et adaptés à la culture du fonio (Saidou et al., 2014 ; Sekloka et al., 2016). Pour la collecte de ces données, 03 carrés d'observation de un mètre carré ( $1 \mathrm{~m} 2)$ chacune, ont été aléatoirement posés dans chaque parcelle élémentaire (Gueye, 2016). La dose de semence a été prise à l'échelle de toute la parcelle, alors que le nombre de talles a porté sur 08 plants préalablement marqués de façon aléatoire dans chaque carré deux à trois semaines après semis. L'observation participative et le chronométrage lors des différentes opérations (semis, désherbage/sarclage) ont permis de déterminer les durées de chaque opération. Les perceptions « facile ou difficile » de dix producteurs de chaque groupement villageois expérimentateur ont été collectées sur la pénibilité des opérations du semis et de désherbage, en fonction de chaque type de semis.

Tableau 2 : Description des variables

\begin{tabular}{|c|c|c|}
\hline Stades & Variables (unités) & Descriptions \\
\hline \multirow[t]{2}{*}{ Semis } & Dose de semis $(\mathrm{kg} / \mathrm{ha})$ & Quantité de semence utilisée par unité de surface \\
\hline & Durée du semis (h/ha) & Durée du semis par unité de surface \\
\hline \multirow[t]{4}{*}{$\begin{array}{l}\text { Levée- } \\
\text { végétative }\end{array}$} & Date de levée (jas) & $\begin{array}{l}\text { Nombre de jours après semis (jas) où } 50 \% \text { des } \\
\text { plants ont levé sur l'ensemble des carrés }\end{array}$ \\
\hline & $\begin{array}{l}\text { Densité de plant } \\
\left(\text { plants } / \mathrm{m}^{2}\right)\end{array}$ & $\begin{array}{l}\text { nombre de plant par unité de surface, comptés } \\
\text { environs } 10 \text { à } 15 \text { jours après la levée }\end{array}$ \\
\hline & $\begin{array}{c}\text { Durée au désherbage } \\
(\mathrm{h} / \mathrm{ha})\end{array}$ & Durée du semis par unité de surface \\
\hline & $\begin{array}{l}\text { Nombre de talles par } \\
\text { plant }\end{array}$ & $\begin{array}{l}\text { Nombre de talles par plant comptées à intervalle } \\
\text { de } 7 \text { jours à partir du } 25^{\text {eme }} \text { jour après semis }\end{array}$ \\
\hline Epiaison & $\begin{array}{l}\text { Résistance à la } \\
\text { verse }(\%)\end{array}$ & $\begin{array}{l}\text { nombre de plant versé suite à une intempérie } \\
\text { (vent violent, orage, pluie) dans chaque carré }\end{array}$ \\
\hline
\end{tabular}




\begin{tabular}{lcl}
\hline \multirow{2}{*}{$\begin{array}{l}\text { Maturatio } \\
\text { n-récolte }\end{array}$} & $\begin{array}{c}\text { Rendement en grain } \\
(\mathrm{kg} / \mathrm{ha})\end{array}$ & $\begin{array}{l}\text { Poids des grains après battage/vannage rapporté } \\
\text { à l'hectare }\end{array}$ \\
\cline { 2 - 3 } & $\begin{array}{c}\text { Rendement en paille } \\
\text { fraîche et sèche }(\mathrm{kg} / \mathrm{ha})\end{array}$ & $\begin{array}{l}\text { Rapport du poids des gerbes fraiches et sèches } \\
\text { par l'hectare }\end{array}$ \\
\hline
\end{tabular}

\subsection{Analyses statistiques des données}

Les données collectées ont été saisies à l'aide du logiciel Excel 2010. Il a été aussi utilisé pour la gestion de la base de données et la représentation des courbes d'évolution de talles. Les analyses statistiques ont été effectuées grâce au logiciel R version 3.1.3 (R Core Team, 2015). Pour les variables quantitatives, les statistiques descriptives (moyennes, écart-types et coefficients de variation) ont été déterminées d'une part, suivies des analyses de variance (ANOVA) après vérification de la normalité et de l'homogénéité des variances d'autre part. Lorsqu'une différence significative est observée, l'analyse de variance a été complétée par le test de Tukey pour la comparaison deux à deux des moyennes au seuil de 5\%. Pour les données qualitatives relatives aux perceptions paysannes sur les opérations de semis et de désherbage, des fréquences relatives des modalités ont été calculées. Les tests d'indépendance de Khi-deux ont été réalisés pour évaluer la relation éventuelle entre les semis en ligne et à volée quant aux perceptions sur les opérations du semis et de désherbage.

\section{Résultats}

\subsection{Dose de semis, levée et densité de plants en fonction des types de semis}

La date de levée des plants n'a pas montré pas une différence significative entre les différents types de semis (Tableau 3). Dans l'ensemble, les plants ont mis 3 à 4 jours après semis pour lever.

Cependant, la quantité de semence utilisée et densité de plants ont révélé de simple voire très forte différences significatives entre types de semis ( $\mathrm{P}<0,05$ ou $\mathrm{P}<0,001)$. Les quantités de semences utilisées et densité de plant pour les semis en ligne ont été nettement supérieures voire le double des semis ordinaires à la volée (Tableau 3 ).

Tableau 3: Dose, date de levée et la densité de plants en fonction des types de semis

\begin{tabular}{|c|c|c|c|}
\hline Type de semis & $\begin{array}{l}\text { dose } \\
\text { semis }(k g / h a)\end{array}$ & de date_levée(jas) & Densité ( plants $/ \mathrm{m}^{2}$ ) \\
\hline SLC25 & $99,45 \quad b$ & 3,4 & $8212,285 \mathrm{~b}$ \\
\hline SLC30 & $81,68 \quad b$ & 3,4 & $7341,995 \mathrm{ab}$ \\
\hline SOV & 48,83 a & 3,5 & 3686,565 a \\
\hline Moyenne & 76,65 & 3,43 & 6413,615 \\
\hline $\mathrm{CV}(\%)$ & 46,18 & 14,68 & 34,34 \\
\hline
\end{tabular}




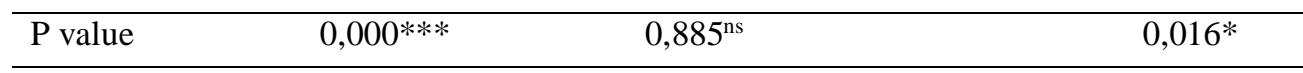

Les valeurs portant les mêmes lettres dans la même colonne sont statistiquement identiques au seuil de 5\%, ns : non significatif ; * significatif, *** : très hautement significatif

\subsection{Durées au semis et au désherbage en fonction des types de semis}

Les types de semis présentent une différence fortement significative pour les durées passées au semis et au désherbage (Tableau 4). Ainsi, l'opération du semis a nécessité plus de temps en ligne qu'à la volée. Le temps passé pour un semis en ligne d'une parcelle a valu sept à neuf fois le temps pour un semis à la volée. Par contre, l'opération du désherbage a été plus rapide dans le semis en ligne comparativement à la volée.

Tableau 4: Durée au semis et au désherbage dans chaque type de semis

\begin{tabular}{ccccc}
\hline Type de semis & \multicolumn{2}{c}{ Durée au semis $(h / h a)$} & \multicolumn{2}{c}{ Durée au désherbage $(h / h a)$} \\
\hline SLC25 & 35,96 & $\mathrm{~b}$ & 28,97 & $\mathrm{ab}$ \\
SLC30 & 27,3 & $\mathrm{~b}$ & 26,22 & $\mathrm{a}$ \\
SOV & 4,05 & $\mathrm{a}$ & 39,26 & $\mathrm{~b}$ \\
Moyenne & 22,44 & 31,49 & \\
CV $(\%)$ & 1,52 & 35,02 & \\
P value & $0,000^{* * *}$ & $0,023^{*}$ & \\
\hline
\end{tabular}

Semis ordinaire à la volée (SOV), en ligne continue à $25 \mathrm{~cm}$ (SLC25) à 30cm (SLC30).

Pour chaque variable, les valeurs portant les mêmes lettres sont statistiquement identiques au seuil de 5\%; * significatif, ***: très hautement significatif

\subsection{Aptitude au tallage des plants sous les types de semis}

Le nombre de talle a varié d'environ 2 à 7 talles par plant dans l'ensemble des types et écartements de semis. Les courbes d'évolution ont montré que les plants du fonio ont développé plus de talles dans le cas du semis à la volée qu'au niveau des semis en ligne continue. En plus, les plants semés en ligne avec un écartement réduit (SLC25) ont des vigueurs plus faibles au tallage (Figure 2).

Tableau 5 : Résistance à la verse des plants

\begin{tabular}{llcl}
\hline & & Nombre de plants versés/ $\mathbf{~ m}^{\mathbf{2}}$ \\
\hline Type_semis & Phase végétative & Phase d'épiaison & Phase de maturation \\
\hline SLC25 & $7,83 \pm 2,96$ & $53,87 \pm 19,98$ & $106,87 \pm 42,43$ \\
SLC30 & $6,77 \pm 2,75$ & $63,00 \pm 14,38$ & $104,63 \pm 28,95$ \\
SOV & $6,57 \pm 2,85$ & $49,60 \pm 15,57$ & $78,30 \pm 27,06$ \\
Moyenne & $7,06 \pm 2,85$ & $55,49 \pm 16,64$ & $96,60 \pm 32,81$ \\
CV $(\%)$ & 39,84 & 30,99 & 36,16 \\
P & $0,57^{\mathrm{ns}}$ & $0,21^{\mathrm{ns}}$ & $0,13^{\mathrm{ns}}$ \\
\hline
\end{tabular}

Semis ordinaire à la volée (SOV), en ligne continue à $25 \mathrm{~cm}$ (SLC25) à $30 \mathrm{~cm}$ (SLC30), ns : non significatif. 


\subsection{Effets des types et écartements de semis sur les rendements}

Les rendements en grain et en biomasse ont été tous en faveur des semis en ligne continue. Toutefois, seul le rendement en grain a permis de discriminer significativement les types et écartements de semis $(p<0,05)$ (Tableau 6). Ainsi, le rendement en grain du fonio a été significativement meilleur dans les semis en ligne continue comparativement au semis à la volée. Un surplus de rendement de plus de $150 \mathrm{~kg} / \mathrm{ha}$ voire près de $200 \mathrm{~kg} / \mathrm{ha}$ a été obtenu avec le semis en ligne par rapport au celui à la volée bien que le rendement moyen en grain pour l'ensemble a été de l'ordre d'une demi tonne à l'hectare soit $548 \mathrm{~kg} / \mathrm{ha}$ (tableau 6).

Tableau 6: Rendements en paille fraiche et sèche du fonio

\begin{tabular}{|c|c|c|c|c|c|c|c|}
\hline Type semis & $\begin{array}{l}\text { Rdt_gr: } \\
(\mathrm{kg} / \mathrm{ha})\end{array}$ & & & \multicolumn{2}{|l|}{$\begin{array}{l}\text { Rbiom_p } \\
\mathbf{f} \\
(k g / h a)\end{array}$} & Rbiom_p & \\
\hline SLC25 & 625,56 & $\pm 238,7$ & $\mathrm{~b}$ & 4922,22 & $\pm 1297,46$ & 2829,63 & $\pm 998,49$ \\
\hline SLC30 & 590,56 & $\pm 29,45$ & $a b$ & 4414,82 & $\pm 1351,31$ & 2542,59 & $\pm 877,82$ \\
\hline SOV & 430,37 & $\begin{array}{l} \pm 194,8 \\
7\end{array}$ & $\mathrm{a}$ & 4264,82 & $\pm 1661,42$ & 2414,82 & $\pm 914,75$ \\
\hline Moyenne & 548,83 & & & 4533,95 & & 2595,68 & \\
\hline $\mathrm{CV}(\%)$ & 42,61 & & & 31,90 & & 35,85 & \\
\hline $\mathrm{P}$ & $0,025^{*}$ & & & $0,367^{\mathrm{ns}}$ & & $0,399^{\mathrm{ns}}$ & \\
\hline
\end{tabular}

Semis ordinaire à la volée (SOV), en ligne continue à $25 \mathrm{~cm}$ (SLC25) à $30 \mathrm{~cm}$ (SLC30), Rendement en grain (Rdt_grain), en paille fraîche (Rbiom_pf) et en paille sèche (Rbiom_pf), Pour chaque variable, les valeurs portant les mêmes lettres sont statistiquement identiques au seuil de 5\%* : significatif ;ns : non significatif

\subsection{Perceptions des producteurs sur les opérations de semis et de désherbage}

Le test d'indépendance de Khi-deux a révélé que les appréciations des producteurs sur l'exécution des opérations de semis en ligne et à la volée n'ont pas une relation significative (tableau 7). Cependant, la plupart des producteurs interviewés $(85,38 \%)$ ont soutenu à la fois que le semis ordinaire à la volée est facile à exécuter alors que le semis en ligne continue est difficile (tableau 7).

Tableau 7: Perceptions des producteurs sur l'opération de semis

\section{Semis en ligne continue}

\begin{tabular}{cccc}
\hline & & difficile & facile \\
Semis à la volée & difficile & 8,46 & 0,00 \\
& facile & 85,38 & 6,15 \\
\hline & $\mathrm{X}^{2}=0,788 ; \mathrm{ddl}=1 ; \mathrm{P}=0,3747^{\mathrm{ns}}$ \\
\hline
\end{tabular}


Par ailleurs, pour le désherbage, les résultats du test de Khi-deux ont montré une liaison hautement significative entre les perceptions paysannes (tableau 8). Ainsi, plus de la moitié $(56,4 \%)$ des producteurs interviewés ont perçu que le désherbage est plus facile dans le cas du semis en ligne continue que dans le cas du semis à la volée, contre moins d'un cinquième $(17,5 \%)$ estimant le contraire (Tableau 8).

Tableau 8: Perceptions paysannes sur l'opération de désherbage

\begin{tabular}{cccc}
\hline & & \multicolumn{2}{c}{ Désherbage (semis en ligne continue) } \\
\hline & & difficile & facile \\
Désherbage & difficile & 19,1 & 56,4 \\
(Semis à la volée) & facile & 17,3 & 7,3 \\
\hline & $\mathrm{X}^{2}=17,88 ; \mathrm{ddl}=1 ;$ & $\mathrm{P}=0,000 * * *$ \\
\hline
\end{tabular}

\section{Discussion}

La maitrise et la bonne gestion des pratiques et techniques culturales sont fondamentales pour exploiter au mieux le potentiel génétique des cultures, et par ricochet une bonne expression des performances agronomiques.

Dans l'ensemble, les plants ont mis 3 à 4 jours après semis pour germer. Ces résultats sont en conformité à ceux de Cissé (1975); Vodouhè et al., (2003) qui avaient montré que le fonio germe habituellement en $2-4$ jours après le semis.

Pour la dose de semis, les quantités de semences utilisées pour le semis en ligne ont été nettement supérieures voire le double du semis ordinaire à la volée. Egalement, une corrélation positive s'établit entre la dose de semis et la densité de plant. Ainsi, les parcelles semées en ligne consommant de fortes doses de semences ont enregistré les densités de plants les plus élevés. Ces résultats sont en désaccord avec les résultats habituellement rapportés dans la littérature qui concluaient une meilleure gestion des quantités de semence pour les semis en ligne (Renoux et Dumas, 1905, Gueye, 2016). Les résultats de Gueye (2016) ont révélé que le semis en ligne continue a permis de réduire près de $50 \%$ la dose utilisée pour le semis à la volée. En effet, ce résultat obtenu dans notre étude d'évaluation participative, peut s'expliquer en partie par la latitude laissée aux producteurs qui à priori n'étaient pas préparés à cette opération du semis. Mieux, la dose de semence est aussi fonction de l'écartement de semis. Une réduction de l'écartement entre ligne conduit à une augmentation de ligne de semis sur la parcelle, et par conséquent à une élévation de la quantité de semence et de densité de plant.

Pour la durée du semis, l'opération du semis en ligne a nécessité 7 à 9 fois le temps passé pour la volée. Ce qui pourrait s'expliquer par toute la technicité nécessitant de tracer des lignes de semis et de faire le recouvrement, toute chose auxquelles les producteurs n'étaient pas forcement habitués. Par 
contre, dans le semis à la volée, les semences sont jetées en vrac, sans grand souci d'une répartition uniforme. Quant à la durée passée au désherbage, l'opération a été significativement moins consommatrice de temps dans le semis en ligne qu'à la volée. Ce qui pourrait se traduit par le fait que les plants étant disposés en ligne sur la parcelle, le passage et le désherbage des allées et autour des plants étaient plus faciles, même avec la houe, ce qui n'ait certainement pas facile avec le semis à la volée.

Le nombre de talle a varié d'environ 2 à 7 talles par plant dans l'ensemble des types et écartements de semis. Ces résultats sont similaires à ceux rapportant par Badiane (2006) ; Cruz et al., (2011), qui ont montré que le nombre de talles par plant peut osciller de 2 à 8 . Les résultats ont aussi souligné que les plants ont une vigueur au tallage élevée dans le cas du semis à la volée qu'au niveau des semis en ligne continue. On pourrait également expliquer cette situation par les trop fortes quantités de semences utilisées pour les semis en ligne, ayant conduit à de fortes densités, et par conséquent conduiraient à une compétition intrinsèque entre les plantes. Par ricochet, ce qui pourrait limiter le développement végétatif, en l'occurrence la vigueur au tallage des plants. Tout ceci milite en faveur d'une recherche de méthode optimale pour les semis en ligne.

Les résultats sur la verse ont montré que types et écartements n'ont pas d'effet sur la résistance du fonio à la verse. La sensibilité à la verse augmente avec l'âge des plants. Ceci pourrait s'expliquer par la constitution frêle et la taille des tiges (chaumes) du fonio, et surtout le poids des panicules à maturité créant un déséquilibre au niveau des plants (Dansi et al., 2010).

Les résultats sur le rendement en grain ont montré une valeur moyenne de l'ordre d'une demi-tonne de fonio grain paddy à l'hectare. Ce rendement moyen se situe dans les tendances de 200 et 700 voire $900 \mathrm{~kg} / \mathrm{ha}$ rapportées par les travaux antérieurs (Badiane, 2006, Vodouhè et Achigan Dako, 2006 ; Gueye, 2016). En plus, le rendement en grain a été meilleur dans le semis en ligne continue que dans le cas du semis à la volée. Cette tendance observée va dans le même sens des travaux réalisés par Hinvi et Nonfon (2006) à Koumagou B, un des villages de la même commune de Boukoumbé, et qui ont montré que le rendement du fonio a été meilleur dans le semis en ligne continue. De même, les résultats des travaux de Gueye (2016) au Sénégal, sur les tests de mode de semis, ont souligné que les semis en ligne ont en général donné des rendements significativement supérieurs à ceux obtenus avec le semis à la volée.

Par ailleurs, les perceptions paysannes ont montré que l'opération du semis est plus facile à la volée qu'en ligne tandis que les appréciations contraires ont été notées pour l'opération de désherbage. Une fois encore, ces perceptions confirment les résultats enregistrés sur les durées de ces deux 
opérations. Ces résultats sont en conformité à ceux de Cissé (1975) qui a aussi montré que le semis en ligne permet d'assurer un désherbage facile des lignes.

\section{Conclusion}

Cette étude a permis de faire en vraie grandeur dans la commune de Boukombé, une évaluation participative du semis en ligne continue comparée au semis à la volée. Les résultats ont montré que l'opération du semis a nécessité de manière significative une grande quantité de semence et de temps en ligne qu'à la volée. Contrairement à la volée, le désherbage est possible à la houe et nécessite une courte durée dans les parcelles ensemencées en semis en ligne continue. Les types de semis n'ont eu aucun effet significatif sur la verse des plants. Les rendements en grain ont été discriminés par les types de semis. Des rendements meilleurs ont été obtenus avec les semis en ligne continue avec un surplus dépassant $150 \mathrm{~kg} / \mathrm{ha}$ à près de $200 \mathrm{~kg} / \mathrm{ha}$ par rapport au semis à la volée. Les deux écartements du semis en ligne testés ont présenté globalement des tendances similaires pour l'ensemble des variables évaluées. Les perceptions recueillies ont révélé que l'opération du semis a été plus difficile en ligne qu'à la volée. Le désherbage a été par contre plus facile dans le semis en ligne continue comparativement à la volée. Un semis en ligne amélioré par un outil mécanique équipé de semoir comme alternative à la technique classique de la corde, permettrait d'insuffler une véritable relance à la production du fonio dans la zone.

\section{Remerciements}

Les auteurs remercient les ONG ECLOSIO ex Aide au Développement Gembloux (ADG) et Louvain Coopération (LC) pour leurs soutiens financiers et cordiales collaborations à travers le programme AMSANA.

\section{References:}

1. Adoukonou-Sagbadja, H., Wagner, C., Ordon, F., \& Friedt W. (2010). Reproductive system and molecular phylogenetic relationships of fonio millets (Digitaria spp., Poaceae) with some polyploid wild relatives. Tropical Plant Biology.3 (4) : 240-251.

2. Badiane, M. (2006). Agronomie de la culture du fonio. Document de synthèse de formation, Direction Régionale de l'ANCAR Kolo (Sénégal). 10p.

3. Ballogoun, V. Y. (2013). Systèmes post-récolte, transformation, qualité du fonio et produits dérivés au nord du Bénin. Thèse de Doctorat unique. Faculté des Sciences Agronomique. Université d'Abomey-Calavi. Bénin. 152p

4. Bioversity International, IRRI, \& WARDA (2007). Descriptors for wild and cultivated rice (Oryza spp.). Bioversity International, Rome, 
Italy; International Rice Research Institute, Los Baños, Philippines; WARDA, Africa Rice Center, Cotonou, Benin.72p.

5. Cissé, I.B. (1975). La culture de fonio et quelques aspects écophysiologiques de la plante. Wageningen, Netherlands. 72 p.

6. Cruz, J.F., Béavogui, F., \& Dramé, D. (2011). Le fonio, une céréale africaine. Collection agricultures tropicales en poche. Editions QUAE, Cta, Presses agronomiques de Gembloux. 175p.

7. Dansi, A., Adoukonou-Sagbadja H., \&Vodouhe R. (2010). Diversity, conservation and related wild species of fonio millet (Digitaria spp.) in the northwest of Benin. Genetic Resources and Crop Evolution.57(6):832-834.

8. Gueye, M. (2016). Amélioration des techniques de semis, de fertilisation et de récolte du fonio blanc (Digitaria exilis Stapf ; Poaceae) au Sénégal. Thèse de Doctorat en Biologie, Physiologie et Productions Végétales. Faculté des Sciences et Techniques, Université Cheikh Anta Diop de Dakar (Sénégal). 102p + Annexes.

9. Hinvi, J.C., \& Nonfon, R. C. (2006). Rapport d'activités de suiviappui-conseil des activités de démonstration et de pré-vulgarisation du riz et du fonio. PAMRAD/COGESTION/CONTRAT/0421/05. Centre de Recherches Agricoles Nord, recherche-développement Atacora. $23 p$

10. MAEP (2010). Ministère de l'agriculture de l'élevage et de la pêche / CeRPA

Statistique.http://www.countrystat.org/ben/cont/pxwebquery/ma/053s pd010/fr/vType/quickc

11. Paraïso, A. A., Sossou, A. C. G., Yegbemey, R. N., \& Biaou, G. (2011). Analyse de la rentabilité de la production du fonio (Digitaria exilis.) dans la commune de Boukoumbé au Bénin. J. Rech. Sci. Univ. Lomé (Togo), 13(1) : 27-37

12. $\mathrm{R}$ Core Team (2015). R: A language and environment for statistical computing. R Foundation for Statistical Computing, Vienna, Austria. URL http://www.R-project.org/.

13. Renoux, L., \&Dumas P. (1905). Culture du fonio dans la vallée du Sénégal et du Haut Niger. L'agriculture Pratique des Pays Chauds. Bull. Jard. Col. Nogent. 32 : 357-359.

14. Saidou, S. I., Bakasso, Y., Inoussa, M. M., Zaman-Allah, M., Atta, S., Barnaud, A., Billot C., \& Saadou, M. (2014). Diversité agromorphologique des accessions de fonio [Digitaria Exilis (Kippist.) Stapf.] au Niger. In Int. J. Biol. Chem. Sci. 8(4): 1710-1729

15. Sekloka, E., Kanlindogbe, C., Biaou, S.S.H., Adoukonou-Sagbadja, H., Kora, A., Motouama, T. F., Seidou, M., Zinsou, V.A., Afouda, L., \&Baba-Moussa, L. ( 2016). Agro-morphological Characterization of 
millet fonio accessions (Digitaria spp. Stapf.) collected in commune of Boukoumbé Northwest of Benin. Journal of Plant Breeding and Crop Science. 8(10): 211-222

16. Vodouhè, S.R., \& Achigan Dako, E.G. (2006). Digitaria exilis (Kippist) Stapf. In: Brink, M., Belay, G. (Editors). PROTA (Plant Resources of Tropical Africa / Ressources végétales de l'Afrique tropicale), Wageningen, Netherlands. http://uses.plantnetproject.org/f/index.php?title=Digitaria_exilis_(PR OTA)\&oldid=95268 (09/08/2017)

17. Vodouhe, S. R., Zannou A., \&Achigan Dako E. (2003). Actes du Premier Atelier sur la Diversité Génétique du Fonio (Digitaria exilis) en Afrique de l'Ouest. Conakry, Guinée, du 04 au 06 Août 1998. Institut International des Ressources Phytogénétiques (IPGRI), Rome, Italie. 73p. 